Abstract S16 Table 1 Diagnostic performance of $\% \mathrm{VLFI}$ and ODI $>3 \%$ for detection of SDB in CHF patients

\begin{tabular}{lll}
\hline & \%VLFI & ODI>3\% \\
\hline Sensitivity & 0.53 & 0.97 \\
Specificity & 0.44 & 0.32 \\
Positive predictive value & 0.45 & 0.53 \\
Negative predictive value & 0.51 & 0.94 \\
Positive likelihood ratio & 0.94 & 1.42 \\
Negative likelihood ratio & 1.08 & 0.08 \\
Area under receiver operating characteristic curve & 0.49 & 0.92 \\
\hline
\end{tabular}

Funding This study was funded by the British Heart Foundation.

\section{S17 A PILOT STUDY OF THE PREVALENCE OF SLEEP DISORDERED BREATHING (SDB) AND NOCTURNAL HYPOXIA IN SYMPTOMATIC ADULTS WITH SICKLE CELL DISEASE (SCD) AND ITS RELATIONSHIP WITH DISEASE SEVERITY}

doi:10.1136/thx.2010.150912.17

${ }^{1} \mathrm{P}$ Murphy, ${ }^{2} \mathrm{R}$ Dillon, ${ }^{3} \mathrm{~A} J$ W Williams, ${ }^{2} \mathrm{~J}$ Howard, ${ }^{1} \mathrm{~N}$ Hart. ${ }^{1}$ Guy's \& St Thomas' NHS Foundation Trust and Kings College London NIHR Biomedical Research Centre, London, UK; ' ${ }^{2}$ Department of Haematology, Guy's \& St Thomas' NHS Foundation Trust, London, UK; ${ }^{3}$ Lane Fox Respiratory Unit, Guy's \& St Thomas' NHS Foundation Trust, London, UK

Introduction There are few effective therapies available for the longterm management of the cardiac and renal sequelae of SCD. Identifying reversible factors, which exacerbate disease severity, would facilitate development of new therapies or novel applications of established treatments. Nocturnal hypoxia $(\mathrm{NH})$ merits investigation as a disease modulating factor as it is established that hypoxia promotes polymerisation of sickle haemoglobin and this is reversible with oxygen therapy (Noguchi et al, 1993). Although $\mathrm{NH}$ is common in children with SCD and is associated with poor outcome, similar data for adults with SCD are lacking. This is the first study to determine the prevalence of OSA and $\mathrm{NH}$ and quantify the severity of $\mathrm{NH}$ in adults with SCD. In addition, we investigated the correlation between the degree of $\mathrm{NH}$ and organ dysfunction.

Method Patients attending SCD clinic had an Epworth sleepiness score performed. Patients with either an ESS $\geq 10$ or symptoms suggestive of SDB were offered nocturnal oximetry. Nocturnal oximetry findings were objectively scored and compared with the detailed clinical datasets collected at regular clinic attendances. OSA was defined as $4 \%$ oxygen desaturation index (4\% ODI) of $>10$ events/h and $\mathrm{NH}$ was defined as $>30 \%$ total sleep time (TST) with $\mathrm{SpO}_{2}<90 \%$.

Results 93 patients were screened. 34 had ESS $\geq 10$ or clinical symptoms suggestive of SDB. 22 underwent nocturnal oximetry; mean ESS 12 4 , clinic $\mathrm{SpO}_{2} 96 \pm 4 \%, 4 \%$ ODI $8 \pm 6$ events $/ \mathrm{h}$, nocturnal $\mathrm{SpO}_{2} 91 \pm 4 \%$, \%TST $\mathrm{SpO}_{2}<90 \% 43 \pm 41 \%$. Prevalence of OSA and $\mathrm{NH}$ was $59 \%$. The degree of nocturnal hypoxia was correlated with urine protein:creatinine $(r=-0.35, p=0.02)$, elevated pulmonary artery systolic pressure $(r=-0.71 ; p=0.0001)$ and prevalence of priapism $(p=0.004)$. There was no difference detected in frequency of painful crises or hospital admission in patients with significant $\mathrm{NH}$ compared to those without $\mathrm{NH}$.

Conclusion This small pilot study showed that OSA and NH had a prevalence of $59 \%$ in symptomatic adult SCD patients. These data have demonstrated a correlation between the severity of nocturnal hypoxia and pulmonary hypertension, renal impairment and priapism. These observations have not previously been reported. The strength of these correlations could suggest a causal relation- ship, although this needs to be confirmed in a larger prospective trial. Future studies should investigate the relationship between OSA, nocturnal hypoxia and organ dysfunction and need to be focussed on interventions such as nocturnal oxygen and continuous positive airway pressure.

\section{New assessments in cystic fibrosis S18 LONGITUDINAL ASSESSMENT OF BIOMARKERS FOR CLINICAL TRIALS OF NOVEL THERAPEUTIC AGENTS: THE RUN-IN STUDY}

doi:10.1136/thx.2010.150912.18

${ }^{1} \mathrm{E}$ W F W Alton, ${ }^{2} \mathrm{C}$ Boyd, ${ }^{3} \mathrm{~S}$ Cunningham, ${ }^{1} \mathrm{~J} \mathrm{C}$ Davies, ${ }^{4} \mathrm{~S}$ C Hyde, ${ }^{2} \mathrm{~J}$ A Innes, ${ }^{4} \mathrm{D}$ R Gill, ${ }^{2} \mathrm{~A}$ Greening, ${ }^{1} \mathrm{U}$ Griesenbach, ${ }^{1} \mathrm{~T}$ Higgins, ${ }^{2} \mathrm{D} \mathrm{J}$ Porteous. ${ }^{1}$ Department of Gene Therapy, Imperial College, London, UK; ${ }^{2}$ Western General Hospital, Edinburgh, UK; ${ }^{3}$ The Royal Hospital for Sick Children, Edinburgh, UK; ${ }^{4}$ Gene Medicine Research Group, Oxford University, Oxford, UK

We will be undertaking a phase IIB clinical trial of repeated application of liposome-based gene therapy over a one year period in approximately $100 \mathrm{CF}$ patients (Multidose Trial). In preparation for this, we sought to address two key questions. Firstly, could we define the optimal set of patients in which the therapy could both be delivered (good access to the airways via nebulisation), and in whom any therapeutic effect was measurable (one or more abnormal measures of lung disease). Secondly, in this set of 'can deliver-can measure' patients, which biomarker(s) could be powered to be the primary outcome measure for the trial. To address both questions, we undertook a study (Run-in), cross-sectionally assessing 'can deliver' and longitudinally assessing a large set of candidate biomarkers for 'can measure'.192 patients from age 10 upwards, with $\mathrm{FEV}_{1}>40 \%$ were enrolled at two clinical centres; 154 of these remained in the study after four visits spaced at approximately $4-5$ month intervals. Biomarkers assessed cross-sectionally included radionucleotide deposition scans, CT and mucocilary clearance. Longitudinal biomarkers included a large series of serum, sputum and exhaled breath inflammatory markers, lung physiology, exercise-related assays and quality of life assessment. 12 patients were judged too severe for adequate delivery and were excluded. A shortlist of 4 biomarkers was generated based on a) showing a CF/non-CF difference, b) response to course of intravenous antibiotics, and c) coefficients of variation. These four were matched against the remaining 142 patients, and a further seven patients excluded in whom none of these short listed biomarkers was abnormal. 89 patients ( 3 or 4 biomarkers abnormal) have been definitely included to progress into the Multidose Trial, and a further 46 ( 1 or 2 biomarkers abnormal) are awaiting the final primary outcome selection. The Run-in study has, therefore, been able to a) select a cohort of 'optimal' patients in which to assess gene therapy and b) provide an indication of which may be the more useful biomarkers to use in phase IIB clinical trials of novel therapeutic agents.

\section{S19 REAL TIME PCR IN THE IDENTIFICATION AND MANAGEMENT OF ASPERGILLUS IN CF}

doi:10.1136/thx.2010.150912.19

${ }^{1} \mathrm{C}$ G Baxter, ${ }^{2} \mathrm{~A}$ M Jones, ${ }^{2} \mathrm{~A} K \mathrm{~K}$ Webb, ${ }^{1} \mathrm{D}$ W Denning. ${ }^{1}$ University Hospital of South Manchester, Education and Research Department, Manchester, UK; ${ }^{2}$ Manchester Adult CF Unit, Manchester, UK

Purpose The reported prevalence of Aspergillus fumigatus in $\mathrm{CF}$ sputum varies widely from 12 to $57 \%$. While patients with ABPA are routinely treated with antifungals, it is not know whether colonised 